\title{
Ensino de oftalmologia na graduação médica. Estudo comparativo de aprendizado na oftalmoscopia direta com oftalmoscópio convencional e de campo amplo (Panoptic)
}

\section{Teaching ophthalmology at university medical undergraduate course. Comparative study of direct ophthalmoscopy between conventional ophthalmoscope and wide field ophthalmoscope (panoptic) as evaluation method}

Eduardo de França Damasceno ${ }^{1}$, Nadyr Antonia Pereira Damasceno², Adroaldo de Alencar Costa Filho ${ }^{3}$

\section{Resumo}

Objetivo: Comparação da facilidade de aprendizado na realização da oftalmoscopia direta pelos alunos da graduação médica com o uso do oftalmoscópio convencional e do Panoptic. Métodos: Realizou- se um estudo comparativo da facilidade de aprendizado entre o uso do oftalmoscópio convencional e do panoptic para a realização do exame de fundo de olho de pacientes normais e com determinadas alterações específicas de fundo de olho. Foi usado para tal aferição um questionário formulado pelos autores e respondido pelos alunos da graduação na primeira e na oitava semana do curso. Resultados: Na primeira semana do curso, dos 60 alunos avaliados somente $1.60 \%$ tiveram maior facilidade de manipulação do aparelho com o panoptic, contrastando com $10.35 \%$ com o uso do convencional. Na oitava semana, $43.33 \%$ dos alunos demonstrou maior facilidade de manipulação com o uso do panoptic, contrastando com $17 \%$ com o uso do convencional. O teste estatístico de Kruskal Wallis se revelou de significância expressiva $(\mathrm{p}<0,05)$. Conclusão: $\mathrm{O}$ oftalmoscópio de campo amplo (panoptic) necessita de um tempo de aprendizagem de uso um pouco maior em relação ao convencional, porém após este período facilita o aprendizado da oftalmoscopia direta por melhorar a observação das estruturas fundoscópicas.

Descritores: Oftalmoscopia/métodos; Técnicas de diagnóstico oftalmológico; Educação médica; Questionário

\footnotetext{
${ }^{1}$ Staff médico do Setor de Retina e Vítreo do Hospital Universitário Clementino Fraga Filho da Universidade Federal do Rio de Janeiro - UFRJ - Rio de Janeiro (RJ), Brasil;

${ }^{2}$ Staff médico do Setor de Retina e Vítreo do Hospital Naval Marcílio Dias - Rio de Janeiro (RJ), Brasil;

${ }^{3}$ Professor Adjunto do Hospital Universitário Clementino Fraga Filho da Universidade Federal do Rio de Janeiro - UFRJ - Rio de Janeiro (RJ), Brasil.

Instituição: Hospital Universitário Clementino Fraga Filho da Universidade Federal do Rio de Janeiro - UFRJ - Rio de Janeiro (RJ), Brasil Recebido para publicação em: 14/10/2008 - Aceito para publicação em 23/7/2009
} 


\section{INTRODUÇÃO}

$\mathbf{0}$ estudo de propedêutica de fundo de olho se constitui numa das ferramentas fundamentais para a formação acadêmica, durante o curso de graduação médica. Embora variável na sua grade curricular em relação à carga horária, este tópico pedagógico é de grande utilidade, até mesmo como cabedal semiológico de outras especialidades ${ }^{(1,2)}$. Nesta mesma idéia, quanto ao melhor aproveitamento desta carga horária, as atividades práticas nesta área devem ser incentivadas aos alunos, para serem criadas condições, onde os mesmos busquem ativamente o conhecimento(3).

Esta proposta tem como objetivo avaliar criativamente uma forma de aprendizado mais prático de oftalmologia na graduação médica, tal como o exame de fundoscopia. Ainda mais oportunamente, objetiva-se avaliar a propedêutica de um novo tipo de oftalmoscópio de campo amplo. O oftalmoscópio de campo amplo (panoptic) oferece um campo de observação de $30^{\circ}$, enquanto o convencional apresenta-se com campo de $10^{\circ}$, mesmo com dilatação pupilar.

A literatura médica é pouco rica neste assunto, devido em parte a ser um tema didático da formação universitária médica, bem como sob o aspecto específico mais de técnica semiológica em oftalmologia. Abordando de maneira mais direta, somente há o trabalho de Mc Comiskie et al. em 2004 e outro trabalho de Gill et al. também de 2004 como técnica de "screening" para detecção de retinopatia diabética ${ }^{(4,5)}$. Desde então, é pouco conhecida a diferença técnica entre os dois tipos de oftalmoscópios .

Destarte, é inédita na literatura, a idéia de aproveitar a praticidade do uso do oftalmoscópio de campo amplo (panoptic) no ensino fundamental de oftalmologia.

Assim, torna-se o objetivo deste trabalho avaliar comparativamente a facilidade de aprendizado de oftalmoscopia pelos alunos da graduação médica da Universidade Federal do Rio de Janeiro (UFRJ), utilizando dois aparelhos oftalmoscópios distintos: convencional x panoptic.

\section{Metodos}

Amostragem: 60 alunos da turma do $8^{\circ}$. período do curso de Medicina da Universidade Federal do rio de janeiro(UFRJ), realizado de fevereiro a junho de 2002.
Avaliação por meio de questionário de aprendizado, preenchido por cada estudante após a $1^{\mathrm{a}} \mathrm{e}$ a $8^{a}$ semana de instrução de aulas práticas no ambulatório de oftalmologia.

\section{Oftalmoscópio:}

Allen)

Oftalmoscópio direto convencional (Welch

Oftalmoscópio direto de campo amplo (panoptic - Welch Allen)

Observações técnicas: Todos os alunos foram instruídos e treinados continuamente com ambas as técnicas de oftalmoscopia, simultaneamente, sem que houvesse privilégio do treinamento de uma técnica sobre a outra.

Questionário de avaliação de aprendizado: Baseado em variáveis com graduação qualitativa e nominativa crescente denominada de níveis fácil, moderado e difícil ( segundo a facilidade de observação de sinais de fundo de olho e manipulação dos dois oftalmoscópios) . O auxílio dos professores ou monitores no manejo da fundoscopia seria peça fundamental para acerto final do questionário .

Três subgrupos postulados nos questionários são analisados a seguir :

Manipulação dos oftalmoscópios : Fácil, com focalização imediata sem qualquer ajuda do professor ou mestrando na aula prática. Moderado com ajuda do instrutor no máximo uma ou duas vezes para observação do fundo de olho. Difícil quando não consegue visualizar nem focalizar o fundus ocular sem a ajuda do professor ou monitor .

Visualização de estruturas oculares em pacientes normais: Fácil, conseguindo de imediato visualizar todas as estruturas do fundus ocular. Moderado, ao conseguir visualizar as estruturas somente após ajuda do instrutor. Difícil, quando não consegue observar todas as estruturas mesmo com a ajuda do instrutor .

Em pacientes normais observar as estruturas de mácula como o brilho foveal, vasos retinianos com sua relação normal de calibre artéria/veia e cruzamento arteriovenoso normal, além de bordos, tamanhos e contornos da papila óptica.

Observação de lesões específicas : Fácil, conseguindo de imediato observar todas as lesões do fundus ocular. Moderado, conseguindo visualizar as lesões somente após ajuda do instrutor. Difícil, quando não consegue observar todas as lesões mesmo com a ajuda do instrutor.

A seguir exemplificação do questionário utili- 
Quadro 1

Diagrama de exemplificação do questionário de avaliação de aprendizado

\begin{tabular}{|c|c|c|c|c|c|c|c|}
\hline Subgrupos & Variáveis & Oftalmoscópio & $\begin{array}{c}\text { Pacientes } \\
\text { normais }\end{array}$ & $\begin{array}{c}\text { Retinopatia } \\
\text { diabética }\end{array}$ & DMRI & $\begin{array}{c}\text { Retinose } \\
\text { pigmentosa }\end{array}$ & $\underset{\text { miopia }}{\text { Alta }}$ \\
\hline \multirow{6}{*}{$\begin{array}{l}\text { Manipulação } \\
\text { instrumento }\end{array}$} & \multirow[t]{2}{*}{ Fácil } & Panoptic & & & & & \\
\hline & & Convencional & & & & & \\
\hline & \multirow[t]{2}{*}{ Moderado } & Panoptic & & & & & \\
\hline & & Convencional & & & & & \\
\hline & \multirow[t]{2}{*}{ Difícil } & Panoptic & & & & & \\
\hline & & Convencional & & & & & \\
\hline \multirow{6}{*}{$\begin{array}{l}\text { Visualização } \\
\text { de estruturas }\end{array}$} & \multirow[t]{2}{*}{ Fácil } & Panoptic & & & & & \\
\hline & & Convencional & & & & & \\
\hline & \multirow[t]{2}{*}{ Moderado } & Panoptic & & & & & \\
\hline & & Convencional & & & & & \\
\hline & \multirow[t]{2}{*}{ Difícil } & Panoptic & & & & & \\
\hline & & Convencional & & & & & \\
\hline \multirow{6}{*}{$\begin{array}{l}\text { Lesões } \\
\text { específicas }\end{array}$} & \multirow[t]{2}{*}{ Fácil } & Panoptic & & & & & \\
\hline & & Convencional & & & & & \\
\hline & \multirow[t]{2}{*}{ Moderado } & Panoptic & & & & & \\
\hline & & Convencional & & & & & \\
\hline & \multirow[t]{2}{*}{ Difícil } & Panoptic & & & & & \\
\hline & & Convencional & & & & & \\
\hline
\end{tabular}

NT: O mesmo questionário é repetido no início ( $1^{\text {a }}$ semana) e ao final do curso prático na oitava semana

zado pelos alunos (Quadro 1).

Estas variáveis foram dispostas em subgrupos nos questionários, de forma sempre a favorecer a análise comparativa entre os dois tipos de oftalmoscópios .

As morbidades escolhidas para representar as aulas práticas de fundoscopia foram selecionadas segundo a importância para a formação acadêmica do graduando, (retinopatia diabética e DMRI, formas secas ou úmidas), bem como também para servir de quesitos técnicos para avaliação crítica entre os dois oftalmoscópios (retinose pigmentar avançada e alta miopia).

Teste estatístico não paramétrico avaliado: Kruskal Wallis, com nível de significância de 5\% para aprovação .

\section{Resultados}

Os dados são aplicados em tabelas comparativas $\left(1^{\mathrm{a}}\right.$ e $8^{\mathrm{a}}$ semana) que refletem o decorrer do tempo de aplicação das aulas práticas de fundo de olho (Tabelas 1 e 2).

Observando-se as percentagens, nota-se uma típica tendência dos alunos após a primeira semana de aprendizado da necessidade do auxílio e orientação de professores e monitores para uso do oftalmoscópio (índices das variáveis fácil, moderado e difícil nos três subgrupos pesquisados), havendo também uma discreta tendência favorável ao oftalmoscópio convencional.

Ao final da primeira semana, os alunos apresentaram uma maior facilidade de aprendizado com o uso 
Tabela 1

Avaliação comparativa entre oftalmoscopia direta com oftalmoscópio convencional e panoptic - 1a semana de curso prático de graduação

\begin{tabular}{|c|c|c|c|c|c|c|c|c|c|}
\hline Subgrupos & Variáveis & Oftalmoscópio & $\begin{array}{l}\text { Pacientes } \\
\text { normais }\end{array}$ & $\begin{array}{l}\text { Retinopatia } \\
\text { diabética }\end{array}$ & DMRI & $\begin{array}{c}\text { Retinose } \\
\text { pigmentosa }\end{array}$ & $\begin{array}{l}\text { Alta } \\
\text { miopia }\end{array}$ & Total & $\%$ \\
\hline \multirow{6}{*}{$\begin{array}{l}\text { Manipulação } \\
\text { instrumento }\end{array}$} & \multirow[t]{2}{*}{ fácil } & panoptic & 1 & 1 & 1 & 0 & 2 & 5 & $1,60 \%$ \\
\hline & & convencional & 12 & 2 & 6 & 3 & 8 & 31 & $\underline{10,35}$ \\
\hline & \multirow[t]{2}{*}{ moderado } & panoptic & 20 & 11 & 16 & 2 & 25 & 74 & $24,60 \%$ \\
\hline & & convencional & 7 & 14 & 13 & 9 & 13 & 56 & $18,60 \%$ \\
\hline & \multirow[t]{2}{*}{ difícil } & panoptic & 17 & 15 & 21 & 26 & 5 & 84 & $\underline{28 \%}$ \\
\hline & & convencional & 3 & 17 & 3 & 20 & 7 & 50 & $16,66 \%$ \\
\hline \multirow{6}{*}{$\begin{array}{l}\text { Visualização } \\
\text { estruturas } \\
\text { normais }\end{array}$} & \multirow{2}{*}{ fácil } & panoptic & 1 & $*$ & $*$ & $*$ & $*$ & 1 & $1,60 \%$ \\
\hline & & convencional & 12 & $*$ & $*$ & $*$ & $*$ & 12 & $\underline{20,00 \%}$ \\
\hline & \multirow[t]{2}{*}{ moderado } & panoptic & 20 & $*$ & $*$ & $*$ & $*$ & 20 & $\overline{33,33 \%}$ \\
\hline & & convencional & 7 & $*$ & $*$ & $*$ & $*$ & 7 & $\overline{11,66 \%}$ \\
\hline & \multirow[t]{2}{*}{ difícil } & panoptic & 17 & $*$ & $*$ & $*$ & $*$ & 17 & $28,33 \%$ \\
\hline & & convencional & 3 & $*$ & $*$ & $*$ & $*$ & 3 & $5 \%$ \\
\hline \multirow{6}{*}{$\begin{array}{l}\text { Lesões } \\
\text { específicas }\end{array}$} & \multirow[t]{2}{*}{ fácil } & panoptic & $*$ & 1 & 1 & 0 & 1 & 3 & $1,25 \%$ \\
\hline & & convencional & $*$ & 2 & 6 & 3 & 5 & 16 & $6,66 \%$ \\
\hline & \multirow[t]{2}{*}{ moderado } & panoptic & $*$ & 17 & 11 & 7 & 22 & 57 & $23,75 \%$ \\
\hline & & convencional & $*$ & 10 & 16 & 8 & 14 & 48 & $20 \%$ \\
\hline & \multirow[t]{2}{*}{ difícil } & panoptic & $*$ & 22 & 18 & 24 & 7 & 71 & $29,58 \%$ \\
\hline & & convencional & $*$ & 8 & 8 & 18 & 11 & 45 & $18,75 \%$ \\
\hline
\end{tabular}

NT: Respostas dos acadêmicos de Medicina após a $1^{\mathrm{a}}$ semana do curso prático de oftalmologia;

* indicando dados não referentes a alocação específica nesta parte da tabela; Dados sublinhados e em negrito revelando os valores mais expressivos em percentuais

Tabela 2

Avaliação comparativa entre oftalmoscopia direta com oftalmoscópio convencional e panoptic $-8^{\text {a }}$ semana de curso prático de graduação

\begin{tabular}{|c|c|c|c|c|c|c|c|c|c|}
\hline Subgrupos & Variáveis & Oftalmoscópio & $\begin{array}{c}\text { Pacientes } \\
\text { normais }\end{array}$ & $\begin{array}{c}\text { Retinopatia } \\
\text { diabética }\end{array}$ & DMRI & $\begin{array}{c}\text { Retinose } \\
\text { pigmentosa }\end{array}$ & $\begin{array}{c}\text { Alta } \\
\text { miopia }\end{array}$ & Total & $\%$ \\
\hline \multirow{6}{*}{$\begin{array}{r}\text { Manipulação } \\
\text { instrumento }\end{array}$} & \multirow[t]{2}{*}{ fácil } & panoptic & 35 & 28 & 37 & 14 & 16 & 130 & $43,33 \%$ \\
\hline & & convencional & 13 & 14 & 14 & 3 & 7 & 51 & $17,00 \%$ \\
\hline & \multirow[t]{2}{*}{ moderado } & panoptic & 10 & 8 & 3 & 15 & 14 & 50 & $16,66 \%$ \\
\hline & & convencional & 2 & 8 & 2 & 11 & 9 & 32 & $10,66 \%$ \\
\hline & \multirow[t]{2}{*}{ difícil } & panoptic & 0 & 0 & 1 & 5 & 4 & 10 & $3,33 \%$ \\
\hline & & convencional & 0 & 2 & 3 & 12 & 10 & 27 & $9,00 \%$ \\
\hline \multirow{6}{*}{$\begin{array}{l}\text { Visualização } \\
\text { estruturas } \\
\text { normais }\end{array}$} & \multirow[t]{2}{*}{ fácil } & panoptic & 31 & $*$ & $*$ & $*$ & $*$ & 31 & $51,66 \%$ \\
\hline & & convencional & 11 & $*$ & $*$ & $*$ & $*$ & 11 & $18,33 \%$ \\
\hline & \multirow[t]{2}{*}{ moderado } & panoptic & 12 & $*$ & $*$ & $*$ & $*$ & 12 & $20 \%$ \\
\hline & & convencional & 5 & $*$ & $*$ & $*$ & $*$ & 5 & $8,33 \%$ \\
\hline & \multirow[t]{2}{*}{ difícil } & panoptic & 0 & $*$ & $*$ & $*$ & $*$ & 0 & 0 \\
\hline & & convencional & 1 & $*$ & $*$ & $*$ & $*$ & 1 & $1,66 \%$ \\
\hline \multirow{6}{*}{$\begin{array}{l}\text { Lesões } \\
\text { específicas }\end{array}$} & \multirow[t]{2}{*}{ fácil } & panoptic & $*$ & $29^{(94,5 \%)}$ & $31^{(61,30 \%)}$ & $11(45.4 \%)$ & $14(50 \%)$ & 85 & $35,41 \%$ \\
\hline & & convencional & $*$ & $2(\%)$ & $12^{(\%)}$ & $5(\%)$ & $7(\%)$ & 26 & $10,83 \%$ \\
\hline & \multirow[t]{2}{*}{ moderado } & panoptic & $*$ & $17^{[47,2 \%]}$ & $7[28,6 \%]$ & $13[31,8 \%]$ & $11^{[10,2 \%]}$ & 48 & $20 \%$ \\
\hline & & convencional & $*$ & $8^{[\%]}$ & $5^{[\%]}$ & $9[\%]$ & $10^{[\%]}$ & 32 & $13,33 \%$ \\
\hline & \multirow[t]{2}{*}{ difícil } & panoptic & $*$ & 2 & 0 & 5 & 5 & 12 & $5 \%$ \\
\hline & & convencional & $*$ & 2 & 5 & 17 & 13 & 37 & $15,41 \%$ \\
\hline
\end{tabular}

NT: Respostas dos acadêmicos de Medicina após a $8^{\text {a }}$ semana do curso prático de oftalmologia;

(*) indicando dados não referentes a alocação específica nesta parte da tabela. Dados sublinhados e em negrito revelando os valores mais expressivos em percentuais 
do oftalmoscópio convencional. Após o auxílio com os professores e monitores, houve uma maior facilidade de aprendizado com o panoptic. Demonstrando que no início do treinamento, os alunos tiveram uma dificuldade maior com o panoptic.

Para os alunos com aprendizado mais difícil, o panoptic ofereceu maior dificuldade nesse período.

Após oito semanas de aprendizado, é visível o maior treinamento dos alunos quase não necessitando mais de auxílio de monitores. Notório, também, é a preferência dos acadêmicos pelo oftalmoscópio de campo amplo (panoptic), havendo muitos dos alunos manifestado interesse em adquiri-lo.

Após a $8^{\text {a }}$ semana, com maior treinamento, os alunos tiveram maior facilidade na realização da oftalmoscopia direta com o panoptic, mesmo aqueles que tenham necessitado de um maior auxílio dos monitores e professores. Para aqueles com maior dificuldade de aprendizado houve uma inversão em relação a $1^{\text {a }}$ semana, com maior dificuldade no uso do oftalmoscópio convencional.

Estatística com teste Kruskal Walis ( $\mathrm{p}=0,032)$, considerando-se significativo ao se comparar as duas tabelas, com expressivo favorecimento aos argumentos acima expostos .

Na observação exclusiva de lesões específicas, os índices das morbidades escolhidas para uma maior análise crítica entre os dois oftalmoscópios pesquisados, na alta miopia e retinose pigmentosa; não houve as expressividades esperadas, sendo os percentuais distribuídos mais regularmente dentre as variáveis: fácil, moderada e difícil.

\section{Discussão}

Conforme relatado no trabalho de Mc Comiskie et al. ${ }^{(4)}$, a vantagem da observação com um campo amplo ao oftalmoscópio é uma prerrogativa valiosa, sugerindo até mesmo a possibilidade de observação de características de topografia da papila óptica na semiologia glaucomatosa sem dilatação pupilar. Corroborou-se esta hipótese, ao se manifestar a maior preferência dos acadêmicos na observação do fundo de olho com o oftalmoscópio panoptic, confirmado com a significância do teste estatístico .

A curva de aprendizado também foi decisiva, ao se revelarem os dados tabulares entre a $1^{\text {a }}$ semana e a $8^{a}$ semana (Tabelas 1 e 2), somente sendo ainda pouco caracterizada nas morbidades escolhidas para uma análise crítica da dificuldade entre os dois oftalmoscópios: alta miopia e retinose pigmentosa. A maior dificuldade de adaptação a uma manipulação mais intricada do panoptic pode explicar esta dissonância, como no relato de maior exigência de experiência prática com este tipo de oftalmoscópio em Gill et al ${ }^{(5)}$, na descrição de sua técnica de "screening" para detecção de retinopatia diabética.

As lesões mais periféricas precisaram de maior auxílio dos monitores e professores que as mais centrais e foram mais bem examinadas com o panoptic, após a $8^{\mathrm{a}}$ semana.

A divulgação de artigos envolvendo pesquisa clínica com os alunos de medicina ainda teve o mérito de incentivo à pesquisa científica na graduação médica, prática muito profícua dentre a formação dos acadêmicos ${ }^{(6,7)}$.

A lembrança deste tipo de instrumento como ferramenta de trabalho e ensino se torna uma importante peça de arsenal propedêutico ressaltando as vantagens da oftalmoscopia direta de campo amplo. Novas modalidades de ensino de propedêutica são voga de uma tendência de condições didáticas mais estimulantes para ensino de oftalmologia ${ }^{(2,8-9)}$.

\section{CONCLUSÃo}

O oftalmoscópio de campo amplo (panoptic) necessita de um tempo de aprendizagem de uso um pouco maior em relação ao convencional (efeito aprendizado), porém após este período facilita o entendimento da oftalmoscopia direta por melhorar a observação das estruturas fundoscópicas.

\section{AbStraCt}

Purpose: Comparison of the skill to teach the direct ophthalmoscopy class supported by fellows of medical graduation course using two examination methods: conventional ophthalmoscope and wide field ophthalmoscope (panoptic). Methods: Assessment of the competence to learn using a survey after eye fundus examination with conventional ophthalmoscope and wide field ophthalmoscope (panoptic). The group searched was composed of 60 students of university medical graduation course. The survey was applied during the first and eighth week of ophthalmogical class of medical graduation. The evaluation of competence to learning was token in as needing to help teachers to examination the eye fundus during the class, and the competence to manipulate the ophthalmoscopes. Tables and statical test (Kruskal Wallis) was performed to demonstrate the results analysis. Results: In the first 
week of ophthalmological class, only $1.60 \%$ of the students chose the panoptic ophthalmoscope, while the same score of $10.35 \%$ chosen with the conventional ophthalmoscope. In the eighth week of ophthalmological class the competence score with the panoptic ophthalmoscope raised to $43.33 \%$, while conventional ophthalmoscope score stayed at 17\% . The statistical analysis of Kruskal Wallis test revealed a positive significance $(p<0,05)$. Conclusion: The panoptic wide field ophthalmoscope needed a longer time to learn how to manipulate and observe the eye fundus, although after this time, the panoptic showed more competence and improved the eye fundus examination.

Keywords: Ophthalmoscopy/methods; Diagnostic techniques, ophtalmological; Education, medical; Questionnaires

\section{ReFERÊNCIAS}

1. Marcondes AM, Nastari ER, Macchiaverini Filho N Avaliação discente de um curso de oftalmologia. Rev Bras Educ Med. 2002, 26(3):171-4 .

2. Costa JG. Curso de graduação, perspectivas do ensino da oftalmologia. Med HUPE-UERJ. 1983; 2(1):27-31.
3. Ginguerra MA, Ungaro AB, Villela FF, Kara-José AC, Kara-José N. Aspectos do ensino de graduação em oftalmologia . Arq Bras Oftalmol. 1998, 61(5):546-50 .

4. McComiskie JE, Greer RM, Gole GA. Panoptic versus conventional ophthalmoscope. Clin Experiment Ophthalmol; 2004, 32(3): 238-42.

5. Gill JM, Cole DM, Lebowitz HM, Diamond JJ. Accuracy of screening for diabetic retinopathy by family physicians. Ann Fam Med; 2004, 2(3): 218-20.

6. Kara José AC, Passos LB, Kara José FC, Kara José N. Ensino extracurricular em Oftalmologia: grupos de estudos / ligas de alunos de graduação . Rev Bras Educ Méd. 2007, 31(2):166-72 .

7. Martins LD, Pandolfo A, Araujo TE, Silveira VM, Darli WS, Taglietti ZR, et al. Informatizaçäo das aulas práticas de oftalmologia: um novo capítulo em didática. Rev Bras Oftalmol. 1999, 58(10):749-52.

8. Lippa LM. Medical student education. Ophthalmology. 2006, 113(5): 890-1.

9. Quillen DA, Harper RA; Haik BG . Medical student education in ophthalmology: crisis and opportunity. Ophthalmology. 2005, 112(11): 1867-8.

Endereço para correspondência:

\section{Eduardo F. Damasceno}

Rua Jangadeiros $n^{\circ} 40$, apto 601 - Ipanema

CEP 22420-010 - Rio de Janeiro - RJ

e-mail: e_damasceno@yahoo.com 